\title{
Stefan Thomas, Joseph Rothmaler, Frauke Hildebrandt, Rebecca Budde \& Stephanie Pigorsch: Partizipation in der Bildungsforschung
}

\author{
Weinheim \& Basel: Beltz Juventa (2020)
}

\author{
Lars Alberth
}

Eingegangen: 4. Juni 2021 / Angenommen: 6. Juni 2021 / Online publiziert: 6. Januar 2022

(C) Der/die Autor(en) 2022

Der zu Besprechung vorliegende Sammelband enthält Beiträge, die Partizipation von Kindern und Jugendlichen entlang von zwei Blickachsen problematisieren: Zum einen geht es um eine Partizipation von Kindern und Jugendlichen an Bildungsforschung, zum zweiten um eine Bildungsforschung über Partizipation dieser Altersgruppen in zwei zentralen institutionellen Feldern: in den sozialpädagogischen Dienstleistungen und in der Schule.

Die erste Blickachse richtet sich am forschungsethischen (und auch rechtlich kodifizierten) Anspruch auf Symmetrie zwischen Forschenden und Beforschten aus und fragt, inwieweit Kindern und Jugendlichen die Möglichkeit zur Partizipation an Bildungsforschung eingeräumt werden soll oder muss. Diesem Problem ist der erste Teil des Sammelbandes gewidmet. Der Band positioniert sich recht deutlich maximalistisch, also im Sinne einer weitestgehenden Einbindung von Kindern und Jugendlichen in die Bildungsforschung. Als normatives Fundament gilt die UNKinderechtskonvention, auf die sich alle vier Texte stützen. So fordert der Beitrag von Stefan Thomas, dass es bei partizipativer Forschung sowohl um Erkenntnisgewinn als auch Erweiterung der Handlungsfähigkeit gehe. Zugleich dokumentiert er sehr klar die Grenzen eines „Peer Research“-Projektes mit unbegleiteten geflüchteten Jugendlichen, das von mehrdimensionalen Übersetzungsprozessen (zwischen Sprachen, generationalen Gruppen, zwischen Expert*innen und Lai*innen), fehlendem Commitment und ökonomischen Transaktionskosten für alle Beteiligten geprägt war. Die Jugendlichen wurden begrenzt auch in die Analyse, Präsentation und Publikation der Forschung eingebunden. Diese nicht-intendierten Nebenfolgen werden zwar forschungsethisch und generational sensibel reflektiert, allerdings wird kein Versuch der Analyse unternommen, an welchen Stellen im Forschungsprozess

Lars Alberth $(\triangle)$

Leuphana Universität Lüneburg, Lüneburg, Deutschland

E-Mail: lars.alberth@leuphana.de 
die Partizipation von Kindern und Jugendlichen sinnvoll erscheint. Implizit gesteht sich der Text dies auch ein, wenn er sich im Anschluss vor allem auf die Darstellung angemessener partizipativer Erhebungsmethoden konzentriert. Auch Virginia Morrow und Jo Boyden teilen prinzipiell diesen maximalistischen Rahmen der Partizipation und gründen ihre forschungsethische Position in der Verknüpfung von Kinderrechten und Well-being. Der Beitrag stellt dann allerdings auch klar Kriterien auf, anhand derer abgewogen werden soll, ob eine Partizipation sinnvoll sei. Sie formulieren zehn Kriterien, die es dann durchaus auch zulassen, sich gegen die Beteiligung von Kindern und Jugendlichen zu entscheiden. E. Kay M. Tisdall weitet die forschungsethische Reflexion partizipativer Methoden durch den Einbezug der Behindertenrechtskonvention aus. Damit solle es gelingen, etablierte Konventionen wie die der Stimme, der Kompetenz und auch der Forschungsethik als „Obsession“ der Kindheitsforschung (S. 116) zu überdenken. Kindheitsforschung müsse anerkennen, dass der aufgerufene kinder- und behindertenrechtliche Rahmen selbst nur als niedrigschwellige Lösung für Partizipation fungieren kann, der selbst durch generationale Asymmetrien geprägt und deshalb emanzipatorisch zu überschreiten sei. Manfred Liebel schließlich plädiert für eine Forschung von Kindern, die es einzig erlaube, kindzentrierte Perspektiven zu entwickeln. Den Erwachsenen komme dabei die Rolle von unterstützenden Mit-Forscher*innen zu. Der Ertrag für Kinder gehe auch hier über die Durchführung von Forschung hinaus, indem es den Kindern Instrumente an die Hand gebe, ihre eigene soziale Position zu stärken und die epistemische Gewalt erwachsenenzentrierter Forschung zu durchbrechen. Nimmt man diese vier Beiträge zusammen, so bleibt allerdings unklar, worauf sich die Idee stützen soll, dass die Kinder und Jugendlichen selbst die Rolle der Forschenden einnehmen sollen. Partizipation mündet hier in der Auflösung der wissenschaftlichen Arbeitsteilung von Beobachtung und Teilnahme. Hier wäre zumindest zu fragen, wie sich die für die Integrität des Forscher*innen-Selbst notwendige Distanzierung vom Forschungsgegenstand bewerkstelligen ließe: Wie lässt sich ein generationales ,going native“ verhindern und der Einfluss von Abhängigkeiten im Feld auf die Erkenntnisproduktion minimieren, wenn die Betroffenen das Forschungsfeld nie verlassen können?

Der zweite analytische Zugriff richtet sich auf die Partizipation von Kindern und Jugendlichen in Sozialpädagogik und Schule - die weiteren Beiträge lassen sich also dezidiert als Bildungsforschung über Partizipation verstehen. Bei den Beiträgen zur Sozialpädagogik sticht heraus, dass vorwiegend pädagogische Institutionen im engeren Sinne untersucht werden: Heime, Internate, Krippen und Kindertagesstätten. Immerhin vergleichen Stephanie Pigorsch und Joseph Rothmaler Kitas mit lokaler Stadtpolitik. Ausgespart bleibt das weitere Feld der Kinder- und Jugendhilfe und des aufsuchenden Kinder- und Jugendschutzes. Nimmt man aber das Versprechen des Partizipationsdiskurses ernst, Demokratiefähigkeit und die Wahrung der Kinderrechte zu gewährleisten, so überrascht diese Einengung auf diese stark heteronomen Kontexte, in denen so etwas wie Citizenship allenfalls formal simuliert werden kann. Der Ertrag von Katrin Peyerls kontrastiven Analyse von Gruppendiskussionen mit Schüler*innen zweier Internate besteht dann auch darin, zu zeigen, dass das selbstwirksame Erleben formalisierter Strukturen repräsentativer Partizipation gerade nicht von ihrer Institutionalisierung selbst, sondern von der Interaktionsge- 
schichte zwischen Internatsleitung und Schülerschaft abhängt. Es ist im übrigen einer von zwei Beiträgen zum Feld der Sozialpädagogik, die sich auf eine Analyse der Aussagen von Kindern und Jugendlichen stützen. In ihrer historischen Rekonstruktion der pädagogische Praxis von Janusz Korczak suchen Urszula MarkowskaManista und Dominika Zakrzweska-Olędzka Anknüpfungspunkte für die pädagogische Arbeit mit vulnerablen Kindern und ein Mittel zur Bewältigung politischer Herausforderungen der Gegenwart. Der Ansatz mündet jedoch sichtbar in einem wohlmeinenden Paternalismus, behütet vom liebevoll „Old Doctor“ genannten Kinderrechtler: Partizipation kann und soll es offensichtlich nicht ohne pädagogische Anleitung geben. Dass es bei der Inanspruchnahme der Partizipationsidee deutlich um die normative Absicherung der pädagogischen Institutionen geht, zeigen dann auch die weiteren Beiträge auf je eigene Weise. Susanne Rechenbach dokumentiert die Entwicklung eines Partizipationsinventars (es handelt sich um eine Erweiterung der Kindergartenskala KES-R) zur Evaluation und Qualitätskontrolle von Kindertageseinrichtungen. Ironischerweise lassen sich mit diesem Inventar Einrichtungen als kinderrechtskonform darstellen, ohne dass die Kinder in der Evaluation gezielt einbezogen werden müssten. Auch der Beitrag von Frauke Hildenbrandt und Bianca Pergande liefert geradezu ein Skript für die Inszenierung von Partizipation: Nachdem diese frühpädagogisch und entwicklungspsychologisch begründet und als Anforderung an den pädagogischen Alltag in Krippen dargelegt wird, folgt eine Art Checkliste zur Überprüfung der partizipativen Qualität von Essensituationen in Krippen. Dass Partizipation zugleich aktiv inszeniert, diskursiv aufgerufen und materiell hergestellt werden muss, konstatieren auch Stephanie Pigorsch und Joseph Rothmaler in ihrer vergleichend angelegten Ethnografie. Sie verweisen im Gegensatz zu den vorhergehenden Arbeiten kritisch darauf, dass ein solches ,,doing participation“ einer Foucaultschen Selbsttechnologie entspreche, die zu einer Pädagogisierung der Partizipanden führe, und fordern, Formen des doing participation gerade auch in nicht-pädagogischen Feldern nachzugehen.

Der dritte Teil zur Schule zeigt sich schließlich erfreulich empirisch und zwei der Beiträge lassen die Kinder und Jugendlichen nun auch ausführlicher zu Wort kommen. André Epp rekonstruiert in biografisch-narrativen Interviews den Einfluss von Biografiearbeit auf berufliche Teilhabe aus der Sicht der Lehrkräfte und der betroffenen Schüler*innen am Übergang zum Berufsleben. Daniela Müller-Kuhn präsentiert die quantitative Auswertung einer explorativen mixed-methods-Studie zu Partizipationswünschen von Schüler*innen und Lehrkräften an Schweizer Volksschulen. Sie zeigt, dass sich die Erfahrung von Partizipationsmöglichkeiten positiv auf die Ausbildung weitergehender Partizipationswünsche auswirken, während das Dienstalter der Lehrkräfte deren Bereitschaft senkt, Schüler*innenpartizipation in der Unterrichtsgestaltung als wünschenswert zu erachten. Malin Kleuker, Ina Rust und Annika Schubotz untersuchen den Zusammenhang von Selbstwirksamkeit und Kompetenzentwicklung im Rahmen des politischen Bildungsprogramms „Dialog macht Schule“. Ihr Fokus ist auf die Ausbildung von Schlüsselkompetenzen der erwachsenen studentischen „Dialogpartner*innen“ gerichtet, die dieses Programm mit den Schüler*innen umsetzen sollen - während die Schüler*innen als Bewährungsprobe für die Professionalisierung zukünftiger Sozialwissenschaftler*innen figurieren. Auch Katjuscha von Werthern stellt mit ihrer Fallstudie zur Elternpartizipation 
an Schulen die Erwachsenen ins Zentrum. Der Autorin zufolge könne es gelingen, mit dem Programm der „Demokratischen Schulentwicklung“ die Beteiligungshürden von Eltern zu minimieren.

Partizipation in der Bildungsforschung - das präsentiert dieser Band nun fast ausschließlich als Herausforderung für die pädagogische Arbeit und die qualitative Bildungsforschung. Das mag so generell formuliert stimmen, doch die hier versammelten Texte zeigen auch, dass Partizipation kaum noch als Angelegenheit der Kinder und Jugendlichen selbst wahrgenommen wird und schon gar nicht mehr zur Analyse von Kindheit und Jugend als Altersgruppe dient. Bei aller kindzentrierten und generational sensiblen Rhetorik in der Ausformulierung forschungsethischer Ansprüche kommt den Kindern und Jugendlichen in diesem Band zur Partizipation eher eine marginale Position zu. Mit Blick auf generationale Asymmetrien ist dieser Bruch zwischen Anspruch und Umsetzung jedoch wesentlich interessanter als die neueste forschungsethische Volte oder die Evaluation partizipationsorientierter Bildungsprogramme.

Funding Open Access funding enabled and organized by Projekt DEAL.

Open Access Dieser Artikel wird unter der Creative Commons Namensnennung 4.0 International Lizenz veröffentlicht, welche die Nutzung, Vervielfältigung, Bearbeitung, Verbreitung und Wiedergabe in jeglichem Medium und Format erlaubt, sofern Sie den/die ursprünglichen Autor(en) und die Quelle ordnungsgemäß nennen, einen Link zur Creative Commons Lizenz beifügen und angeben, ob Änderungen vorgenommen wurden.

Die in diesem Artikel enthaltenen Bilder und sonstiges Drittmaterial unterliegen ebenfalls der genannten Creative Commons Lizenz, sofern sich aus der Abbildungslegende nichts anderes ergibt. Sofern das betreffende Material nicht unter der genannten Creative Commons Lizenz steht und die betreffende Handlung nicht nach gesetzlichen Vorschriften erlaubt ist, ist für die oben aufgeführten Weiterverwendungen des Materials die Einwilligung des jeweiligen Rechteinhabers einzuholen.

Weitere Details zur Lizenz entnehmen Sie bitte der Lizenzinformation auf http://creativecommons.org/ licenses/by/4.0/deed.de. 